\title{
Triglyceride to high-density lipoprotein cholesterol ratio as a predictor of long-term mortality in patients with coronary artery disease after undergoing percutaneous coronary intervention: a retrospective cohort study
}

Xin-Ya Dai ${ }^{1,2+}$, Ying-Ying Zheng ${ }^{1,2^{*}+}$ D, Jun-Nan Tang ${ }^{1,2}$, Xu-Ming Yang ${ }^{3}$, Qian-Qian Guo ${ }^{1,2}$, Jian-Chao Zhang ${ }^{1,2}$, Meng-Die Cheng ${ }^{1,2}$, Feng-Hua Song ${ }^{1,2}$, Zhi-Yu Liu ${ }^{1,2}$, Kai Wang ${ }^{1,2}$, Li-Zhu Jiang ${ }^{1,2}$, Lei Fan ${ }^{1,2}$, Xiao-Ting Yue ${ }^{1,2}$, Yan Bai ${ }^{1,2}$, Zeng-Lei Zhang ${ }^{1,2}$, Ru-Jie Zheng ${ }^{1,2}$ and Jin-Ying Zhang ${ }^{1,2^{*}}$

\begin{abstract}
Background: It has been confirmed that the triglyceride to high-density lipoprotein cholesterol ratio (THR) is associated with insulin resistance and metabolic syndrome. However, to the best of our knowledge, only a few studies with small sample sizes have investigated the relationship between THR and coronary artery disease (CAD). Therefore, we aimed to assess the correlation between the THR and long-term mortality in patients with CAD after undergoing percutaneous coronary intervention $(P C l)$ in our study that enrolled a large number of patients.

Methods: A total of 3269 post-PCl patients with CAD were enrolled in the CORFCHD-ZZ study from January 2013 to December 2017. The mean follow-up time was $37.59 \pm 22.24$ months. Patients were divided into two groups according to their THR value: the lower group (THR $<2.84, n=1232$ ) and the higher group (THR $\geq 2.84, n=2037$ ). The primary endpoint was long-term mortality, including all-cause mortality (ACM) and cardiac mortality (CM). The secondary endpoints were major adverse cardiac events (MACEs) and major adverse cardiac and cerebrovascular events (MACCEs).

Results: In our study, ACM occurred in 124 patients: 30 (2.4\%) in the lower group and 94 (4.6\%) in the higher group $(P=0.002)$. MACEs occurred in 362 patients: $111(9.0 \%)$ in the lower group and $251(12.3 \%)$ in the higher group $(P=0.003)$. The number of MACCEs was 482: $152(12.3 \%)$ in the lower group and $320(15.7 \%)$ in the higher group $(P=0.008)$. Heart failure occurred in 514 patients: $89(7.2 \%)$ in the lower group and $425(20.9 \%)$ in the higher group $(P<0.001)$. Kaplan-Meier analyses showed that elevated THR was significantly related to long-term ACM (logrank, $P=0.044)$ and the occurrence of heart failure (log-rank, $P<0.001)$. Multivariate Cox regression analyses showed that the THR was an independent predictor of long-term ACM (adjusted HR=2.042 [1.264-3.300], $P=0.004$ ) and heart failure (adjusted HR=1.700 [1.347-2.147], $P<0.001$ ).

(Continued on next page)
\end{abstract}

\footnotetext{
* Correspondence: zhengying527@163.com; jyzhang@zzu.edu.cn

${ }^{+}$Xin-Ya Dai and Ying-Ying Zheng contributed equally to this work.

'Department of Cardiology, First Affiliated Hospital of Zhengzhou University,

Zhengzhou 450052, People's Republic of China

Full list of author information is available at the end of the article
}

(c) The Author(s). 2019 Open Access This article is distributed under the terms of the Creative Commons Attribution 4.0 International License (http://creativecommons.org/licenses/by/4.0/), which permits unrestricted use, distribution, and reproduction in any medium, provided you give appropriate credit to the original author(s) and the source, provide a link to the Creative Commons license, and indicate if changes were made. The Creative Commons Public Domain Dedication waiver (http://creativecommons.org/publicdomain/zero/1.0/) applies to the data made available in this article, unless otherwise stated. 
(Continued from previous page)

Conclusions: An increased THR is an independent predictor of long-term ACM and heart failure in post-PCl patients with CAD.

Keywords: Triglyceride to high-density lipoprotein cholesterol ratio, Coronary artery disease, Percutaneous coronary intervention, All-cause mortality

\section{Background}

Coronary artery disease (CAD) has been a major cause of mortality worldwide [1]. Some previous studies have shown that the pathological mechanisms of CAD include the nitric oxide pathway [2], inflammation response [3], estrogen deficiency [4], oxidation [5], thrombosis [6], and lipid metabolism [7]. In a study in 2013, Reiner $\check{Z}$ et al [8] found that $34.7 \%$ of patients with CAD had high triglyceride (TG) levels. Moreover, an ever-growing body of evidence has suggested that TG concentration is significantly associated with an increased risk of CAD [9]. In contrast, high-density lipoprotein cholesterol (HDL-C) levels were inversely related to morbidity in patients with CAD [10]. Furthermore, a study showed that $36.7 \%$ of patients with $\mathrm{CAD}$ had low HDL-C levels [8]. Some previous studies reported that the THR was associated with insulin resistance [11] and metabolic syndrome [12]. However, to our knowledge, there have been only a few studies reporting that the THR had a significant relationship with the extent of the lesion [13], cardiovascular events [14] and long-term ACM [15] in patients with CAD, whereas the sample sizes of these studies were small; none were more than 500 subjects. PCI is a common angioplasty method that is considered a useful therapy for patients with CAD. Some previous studies showed that PCI significantly improved the clinical outcome of patients with CAD $[16,17]$. More recently, Sultani R et al. [15] performed a study in which a high THR independently predicted long-term ACM in 482 CAD patients who underwent coronary angiography without PCI. Therefore, we conducted a study with a larger sample size and aimed to assess whether there was an independent predictive value of an increased THR with regard to long-term mortality in patients with $\mathrm{CAD}$ who underwent PCI.

\section{Methods \\ Study design and population}

A total of 3561 patients were initially enrolled in our study; 292 patients were subsequently eliminated due to unavailable baseline TG or HDL-C data. Ultimately, there were 3269 eligible patients in our study. All of the abovementioned factors are shown in Fig. 1. All of the patients were from the Clinical Outcomes and Risk Factors of Patients with Coronary Heart Disease after PCI

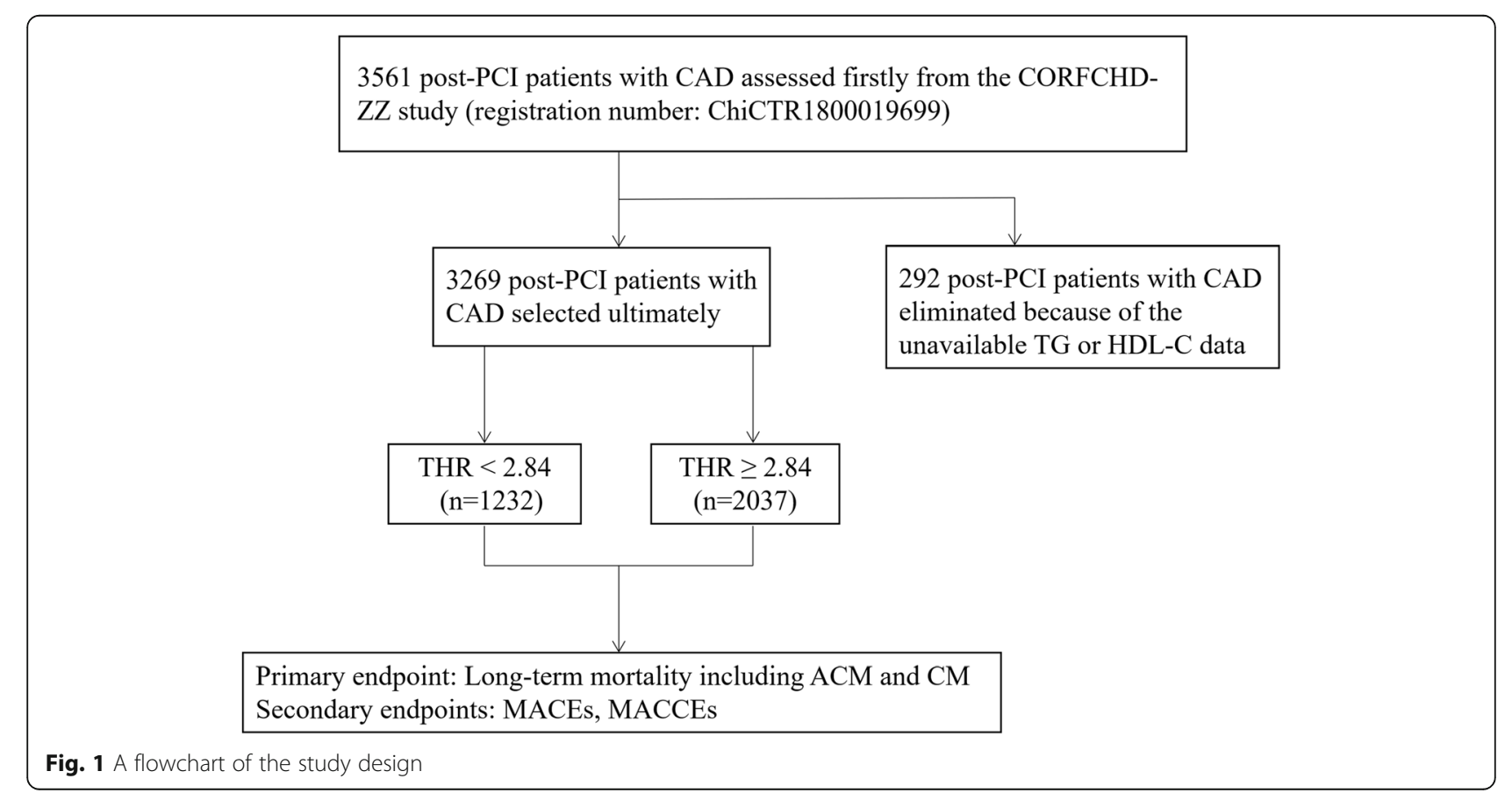


(CORFCHD-ZZ) study, the details of which could be browsed on http://www.chictr.org.cn (registration number: ChiCTR1800019699). The CORFCHD-ZZ study, which was a large, retrospective cohort study, included 3561 post-PCI patients with CAD admitted to the First Affiliated Hospital of Zhengzhou University from January 2013 to December 2017, and its data were obtained from case records and follow-ups. The inclusion criteria for eligibility in the current analysis were as follows: (1) patients aged at least 18 years; (2) at least one instance of coronary artery stenosis $\geq 50 \%$ confirmed by coronary angiography; (3) at least one clinical phenotype of coronary heart disease: stable angina or acute coronary syndrome; and (4) an indispensable and objective check for evidence of myocardial ischemia: positive stress test, FFR $<0.80$ or OCT or IVUS examination suggesting unstable plaque. Patients with the following baseline characteristics were excluded: (1) younger than 18 or older than 80; (2) severe valvular heart disease; (3) severe congenital heart disease; (4) hyperthyroidism, anemia or other high-powered heart disease; (5) pulmonary heart disease; (6) hypertrophic obstructive cardiomyopathy; (7) liver dysfunction (defined as alanine aminotransferase or total bilirubin greater than 3 times the normal upper limit); (8) renal insufficiency (defined as serum creatinine greater than 1.5 times the normal upper limit); or (9) conditions with a high-risk of bleeding, such as thrombocytopenia, blood diseases and other diseases.

\section{Demographic, clinical and laboratory characteristics}

The demographic, clinical and laboratory data were collected from the case records of inpatients at the First Affiliated Hospital of Zhengzhou University. The demographic and clinical data included gender, age, family history of $\mathrm{CAD}$, medical history, hypertension, diabetes, smoking and alcohol consumption. CAD was defined as at least one clinical phenotype of coronary heart disease: stable angina or acute coronary syndrome and at least one instance of coronary artery stenosis $\geq 50 \%$ confirmed by coronary angiography. Hypertension was defined as a previously definitive diagnosis that referred to systolic blood pressure $\geq 140$ $\mathrm{mmHg}$ or diastolic blood pressure $\geq 90 \mathrm{mmHg}$ by using at least three different blood pressure measurements in two disparate healthcare facilities according to the American Heart Association (AHA) Expert Consensus Document [18] or active use of anti-hypertensive drugs. Diabetes was defined as a previous definitive diagnosis that referred to fasting blood-glucose (FPG $\geq 7.0 \mathrm{mmol} / \mathrm{L}$ ), two-hour postprandial glucose (2-h PG) $>11.1 \mathrm{mmol} / \mathrm{L}$ during an oral glucose tolerance test (OGTT) according to the American Diabetes Association (ADA) recommendations [19], or active use of hypoglycemic drugs. Smoking was defined as a previous smoking history or active smoking that referred to smoking day after day or every few days and having ever smoked more than 1 hundred cigarettes [20]. Alcohol drinkers were defined as patients who had consumed alcohol at least once before the study started [21].

The laboratory data included plasma and biochemical parameters such as the levels of TG, HDL-C, total cholesterol (TC), low-density lipoprotein cholesterol (LDL$\mathrm{C})$, blood urea nitrogen (BUN), creatinine $(\mathrm{Cr})$, uric acid (UA), glomerular filtration rate (GFR), glucose (GLU), alanine aminotransferase (ALT), aspartate aminotransferase (AST), and gamma-glutamyl transferase (GGT). After fasting $\geq 12 \mathrm{~h}$, blood samples were collected through a standard venipuncture technique before performing coronary angiography. Then, all blood samples were delivered into the central laboratory at the First Affiliated Hospital of Zhengzhou University.

\section{Endpoints and follow-up}

The primary endpoint was long-term mortality, including $\mathrm{ACM}$ and $\mathrm{CM}$. The secondary endpoints were the composite of major adverse cardiac events (MACEs) and the composite of major adverse cardiac and cerebrovascular events (MACCEs). MACEs were defined as cardiac death, heart failure, bleeding events and readmission. MACCEs were defined as MACEs combined with stroke. Deaths were assumed to result from cardiac causes unless the fatal causes were definitively noncardiac [22]. The heart failure definition complied with the European Society Of Cardiology Guidelines (2016) for heart failure [23]. The definition of a bleeding event was in line with the Bleeding Academic Research Consortium Definition of Bleeding [24]. Readmission was defined as patients admitted to the hospital again, after discharge, due to symptoms or signs of angina pectoris. Stroke was defined as a sudden onset of vertigo, numbness, aphasia, or dysarthria resulting from vascular lesions of the brain, including hemorrhage, embolism, thrombosis, or rupturing aneurysm, and persisting more than 1 day [22].

The follow-up time was from 15 to 50 months, and its mean value was $37.59 \pm 22.24$ months. All investigators underwent standard training on the following: (1) method of follow-up: telephone interviews or office visits; (2) content of follow-up: complying with medical advice, the onset of endpoints and so on. The follow-up was conducted according to the above uniform criterion.

\section{Statistical analysis}

SPSS version 22.0 (SPSS Inc., Chicago, Illinois, United States) was utilized to analyze all data. Continuous variables were presented as the mean \pm standard error and compared using t-tests (for data complying with a normal distribution) or Mann-Whitney U-tests (for data complying with a nonnormal distribution). Categorical variables 
were presented as frequencies and percentages and compared using the chi-square test. ROC curves were utilized to determine the cut-off value of the THR. The KaplanMeier method and the log-rank test were performed to estimate the cumulative incidences of long-term outcomes according to the THR $(<2.84$ and $\geq 2.84)$. Multivariate Cox proportional hazards regression models were conducted to evaluate the independent predictive value of the THR with regard to long-term outcomes. All $P$-values < 0.05 were assumed to be significant.

\section{Results}

\section{Baseline characteristics}

In our study, the cut-off value for the baseline THR was 2.84 according to the analysis of the ROC curve. A total of 3269 post-PCI patients with CAD were divided into two groups according to the THR: the lower group (THR $<2.84, n=1232$ ) and the higher group (THR $\geq$ 2.84, $n=2037)$. In Table 1 , we found that there were significant differences for several variables between the two groups, such as gender, smoking, age, Cr, UA, GLU, TC, TG, and HDL-C (all $P<0.05$ ). However, the following variables were not significantly different between the two groups: family history; hypertension; diabetes; alcohol consumption; medications including calcium channel blocker (CCB), $\beta$-blockers, angiotensin-converting enzyme inhibitor (ACEI) or angiotensin receptor blocker (ARB); systolic blood pressure (SBP); diastolic blood pressure (DBP); BUN; eGFR; ALT; AST; GGT and LDL-C (all $P \geq 0.05$ ).

\section{Outcomes}

As shown in Table 2, the incidence of ACM (2.4\% versus $4.6 \%, P=0.002)$, MACEs $(9.0 \%$ versus $12.3 \%, P=0.003)$, MACCEs $(12.3 \%$ versus $15.7 \%, P=0.008)$ and heart failure $(7.2 \%$ versus $20.9 \%, P<0.001)$ was significantly different between the two groups but CM was not $(1.7 \%$ versus $2.7 \%, P=0.067)$. Furthermore, as shown in Table 3, Fig. 2 and Fig. 3, the Kaplan-Meier analyses

Table 1 Baseline characteristics of patients

\begin{tabular}{|c|c|c|c|c|}
\hline Variables & $\mathrm{THR}<2.84$ & $T H R \geq 2.84$ & $x^{2}$ or $t$ & P-Value \\
\hline Gender, Male, n (\%) & $801(65)$ & $1450(71.2)$ & 13.615 & $<0.001$ \\
\hline Family history, n (\%) & $222(18.1)$ & 389 (19.3) & 0.653 & 0.419 \\
\hline Hypertension, n (\%) & $687(55.8)$ & $1134(55.7)$ & 0.003 & 0.959 \\
\hline Diabetes, n (\%) & $284(23.1)$ & $491(24.1)$ & 0.470 & 0.493 \\
\hline Smoking, n (\%) & $348(28.2)$ & 649 (31.9) & 4.730 & 0.030 \\
\hline Alcohol consumption, n (\%) & $192(15.6)$ & $348(17.1)$ & 1.252 & 0.263 \\
\hline CCB, n (\%) & $229(18.6)$ & $343(16.8)$ & 1.627 & 0.202 \\
\hline$\beta$ - blocker, n (\%) & $595(48.3)$ & $1053(51.7)$ & 3.546 & 0.060 \\
\hline ACEI or ARB,n (\%) & $340(27.6)$ & $579(28.4)$ & 0.260 & 0.610 \\
\hline Age, years & $64.02 \pm 10.51$ & $62.91 \pm 10.62$ & 2.903 & 0.004 \\
\hline $\mathrm{SBP}, \mathrm{mm} \mathrm{Hg}$ & $133.25 \pm 17.59$ & $132.84 \pm 18.07$ & 0.619 & 0.536 \\
\hline $\mathrm{DBP}, \mathrm{mm} \mathrm{Hg}$ & $78.67 \pm 10.78$ & $79.39 \pm 11.41$ & -1.772 & 0.077 \\
\hline BUN, $\mathrm{mmol} / \mathrm{L}$ & $5.77 \pm 5.26$ & $5.76 \pm 4.54$ & 0.076 & 0.939 \\
\hline $\mathrm{Cr}$, umol/L & $70.08 \pm 26.96$ & $74.24 \pm 46.35$ & -2.863 & 0.004 \\
\hline $\mathrm{UA}, \mathrm{mmol} / \mathrm{L}$ & $286.76 \pm 86.07$ & $304.38 \pm 86.73$ & -5.614 & $<0.001$ \\
\hline eGFR,ml/Min & $93.09 \pm 27.59$ & $91.08 \pm 20.99$ & 1.650 & 0.099 \\
\hline GLU, mmol/L & $5.51 \pm 2.05$ & $5.75 \pm 2.50$ & -2.978 & 0.003 \\
\hline$A L T, U / L$ & $25(16-42)$ & $24(16-40)$ & 0.805 & 0.421 \\
\hline AST,U/L & $22(17-32)$ & $21(17-33)$ & 0.126 & 0.990 \\
\hline GGT,U/L & $24(17-40)$ & $25(17-41)$ & 0.257 & 0.797 \\
\hline $\mathrm{TG}, \mathrm{mmol} / \mathrm{L}$ & $2.53(1.33-3.83)$ & $3.59(2.85-4.84)$ & -8.797 & $<0.001$ \\
\hline $\mathrm{TC}, \mathrm{mmol} / \mathrm{L}$ & $2.90(1.42-3.88)$ & $1.56(1.08-2.63)$ & 14.939 & $<0.001$ \\
\hline $\mathrm{HDL}-\mathrm{C}, \mathrm{mmol} / \mathrm{L}$ & $1.01(0.85-1.21)$ & $1.0(0.86-1.18)$ & 2.586 & 0.010 \\
\hline LDL-C, mmol/L & $2.28(1.77-2.86)$ & $2.28(1.81-2.90)$ & -1.342 & 0.180 \\
\hline
\end{tabular}

Data presented as median (interquartile range) or mean \pm SD or $\mathrm{n}(\%)$. Abbreviation: $T H R$ Triglyceride to high-density lipoprotein cholesterol ratio, $C C B$ Calcium channel blocker, $A C E I$ Angiotensin-converting enzyme inhibitor, ARB Angiotensin receptor blocker, SBP Systolic blood pressure, DBP Diastolic blood pressure, $B U N$ Blood urea nitrogen, Cr Creatinine, UA Uric acid, GFR Glomerular filtration rate, GLU Glucose, ALT Alanine aminotransferase, AST Aspartate aminotransferase, GGT Gamma-glutamyl transpeptidase, TG Triglyceride, TC Total cholesterol, HDL-C High-density lipoprotein cholesterol, LDL-C Low-density lipoprotein cholesterol. Note: The boldfaced $P$-Values are statistically different 
Table 2 Outcomes comparison between groups

\begin{tabular}{lllll}
\hline Outcomes & THR $<2.84$ & THR $\geq 2.84$ & $x^{2}$ & $P$-Value \\
\hline ACM, n (\%) & $30(2.4 \%)$ & $94(4.6 \%)$ & 9.993 & $\mathbf{0 . 0 0 2}$ \\
CM, n(\%) & $21(1.7 \%)$ & $55(2.7 \%)$ & 3.35 & 0.067 \\
MACEs, n (\%) & $111(9.0 \%)$ & $251(12.3 \%)$ & 8.553 & $\mathbf{0 . 0 0 3}$ \\
MACCEs, n (\%) & $152(12.3 \%)$ & $320(15.7 \%)$ & 7.065 & $\mathbf{0 . 0 0 8}$ \\
Heart failure, n(\%) & $89(7.2 \%)$ & $425(20.9 \%)$ & 107.785 & $<\mathbf{0 . 0 0 1}$ \\
Bleeding, n (\%) & $13(1.1 \%)$ & $13(0.6 \%)$ & 1.692 & 0.193 \\
readmission, n (\%) & $360(29.2 \%)$ & $605(29.7 \%)$ & 0.085 & 0.771 \\
Stroke, n (\%) & $46(3.7 \%)$ & $77(3.8 \%)$ & 0.005 & 0.946 \\
\hline
\end{tabular}

Abbreviation: THR Triglyceride to high-density lipoprotein cholesterol ratio, ACM All-cause mortality, CM Cardiac mortality, MACEs Major adverse cardiovascular events, MACCEs Major adverse cardiovascular and cerebrovascular events. Note: The boldfaced $P$-Values are statistically different

showed that an elevated THR was significantly related to long-term ACM (log-rank, $P=0.044$ ) and the occurrence of heart failure $(\log$-rank, $P<0.001)$. Multivariate Cox proportional hazards regression models were conducted to evaluate the correlation between the THR and outcomes; the models were adjusted for confounders including age, gender, family history, hypertension, diabetes, smoking, alcohol consumption, $\mathrm{Cr}$, UA, and TC. Patients in the higher THR group had an elevated long-term ACM (adjusted HR = 2.042 [1.264-3.300], $P=$ 0.004 ) and heart failure incidence (adjusted $\mathrm{HR}=1.700$ [1.347-2.147], $P<0.001)$, after being adjusted for several confounders, compared to patients in the lower THR group. Therefore, the increased THR had an independently predictive value for long-term ACM and heart failure. In addition, confounders of long-term ACM and heart failure are shown in Table 4 and Table 5.

\section{Discussion}

In our study, we found that an increased THR was an independent predictor of long-term ACM in post-PCI patients with CAD. Similarly, several studies demonstrated that the THR had a significant relationship with the extent of the lesions [13], cardiovascular events [14] and long-term ACM [15] of CAD, whereas the sample size of these studies was small; none were more than 500. Although a previous study reported that an elevated THR indicated the extent of CAD [13], there were no similar analyses related to the prognosis of CAD aside from our study. Ke Wan et al. [14] demonstrated that an increased THR raised the risk of cardiovascular events in CAD patients; however, compared to our study, the analysis method for determining the THR cut-off value was different, and their study had a smaller sample size (416 enrolled patients). Furthermore, in a study enrolling 482 patients, an increased THR had a significantly predictive value for long-term ACM in CAD patients [15], whereas there was no related comparison of MACCEs between the groups as was done in our study. In addition, a large number of patients were taking medications in our study, and we found that there was no significant difference with respect to the effect of medication use on the THR level in both groups (Table 1). Moreover, the Reduction of Atherothrombosis for Continued Health (REACH) study [25] showed that the use of ACEI/ARBs was not associated with the reduced incidence of adverse endpoints in stable CAD outpatients without HF. Similarly, the use of $\beta$-blockers did not significantly reduce the risk of composite cardiovascular events in CAD patients [26].

In a previous study conducted in a Chinese population, the THR had a powerfully predictive value for insulin resistance but not $\beta$ cell function in patients who had various glucose tolerance statuses [11], and insulin resistance raised the incidence of CAD in patients with type 1 and type 2 diabetes mellitus [27]. Moreover, the THR had a definitive clinical usefulness for indicating the onset of metabolic syndrome [12], which was considered a precursor for the progression of CAD [28]. In addition, the THR was also an independent predictor for the development of arterial stiffness in normotensive patients [29] and was reported to be associated with early signs of structural vascular damage, such as elevated carotid intima-media thickness (CIMT), in children and

Table 3 Incidence of outcomes on multivariate Cox proportional hazards regression models and log-rank test

\begin{tabular}{|c|c|c|c|c|c|c|}
\hline \multirow[t]{2}{*}{ Outcomes } & \multirow{2}{*}{$\begin{array}{l}\mathrm{HR} \\
(95 \% \mathrm{Cl})\end{array}$} & \multirow[t]{2}{*}{ P-Value } & \multirow{2}{*}{$\begin{array}{l}\text { Adjusted HR } \\
(95 \% \mathrm{Cl})^{\mathrm{a}}\end{array}$} & \multirow[t]{2}{*}{$P$-Value } & \multicolumn{2}{|c|}{ log-rank test } \\
\hline & & & & & $x^{2}$ & $P$-Value \\
\hline ACM & $1.521(1.007-2.299)$ & 0.046 & $2.042(1.264-3.300)$ & 0.004 & 4.039 & 0.044 \\
\hline CM & $1.264(0.762-2.094)$ & 0.364 & 1.707 (0.952-3.061) & 0.072 & 0.830 & 0.362 \\
\hline MACES & $1.073(0.857-1.344)$ & 0.540 & $1.143(0.898-1.456)$ & 0.277 & 0.378 & 0.539 \\
\hline Heart failure & 1.912 (1.519-2.406) & $<0.001$ & $1.700(1.347-2.147)$ & $<0.001$ & 32.172 & $<0.001$ \\
\hline MACCES & $0.973(0.801-1.182)$ & 0.784 & $1.023(0.832-1.260)$ & 0.827 & 0.076 & 0.783 \\
\hline
\end{tabular}

Abbreviation: THR Triglyceride to high-density lipoprotein cholesterol ratio, ACM All-cause mortality, CM Cardiac mortality, MACEs Major Adverse cardiovascular events including cardiac death, bleeding events, readmission, MACCEs Major adverse cardiovascular and cerebrovascular events including MACEs and stroke. Note: The boldfaced $P$-Values are statistically different

${ }^{a}$ Adjusted for age, gender, family history, hypertension, diabetes, smoking, alcohol consumption, creatinine, uric acid, and total cholesterol 


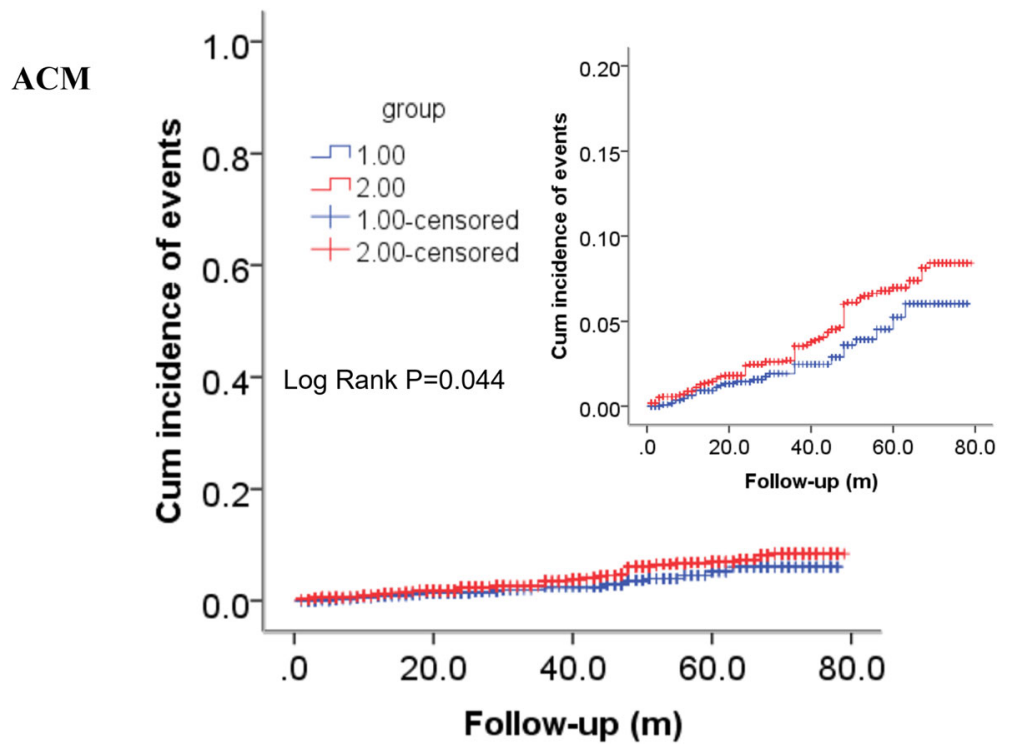

Fig. 2 Cumulative Kaplan-Meier estimates of the time to the first adjudicated occurrence of all-cause mortality

adolescents [30]. Several studies showed that arterial stiffness and elevated CIMT predicted the increased incidence of CAD [31, 32].

There was an inverse correlation between the TG and HDL-C levels in CAD patients; in other words, the HDL-C level in plasma was lower in CAD patients with hypertriglyceridemia [33]. Furthermore, some previous studies demonstrated that high small dense LDL-C levels were significantly related to increased TG concentrations in patients with metabolic syndrome [34] and with reduced HDL-C levels in prediabetic patients [35]. In addition, the THR was also favorable for assessing the presence of small dense LDL-C, whereas it was more difficult and costly to detect small dense LDL-C than THR [36]. Therefore, it was feasible that the elevated THR was utilized as an alternative biomarker indicating increased small dense LDL-C [36], which was significantly associated with the incidence of adverse cardiovascular outcomes in CAD patients [37].

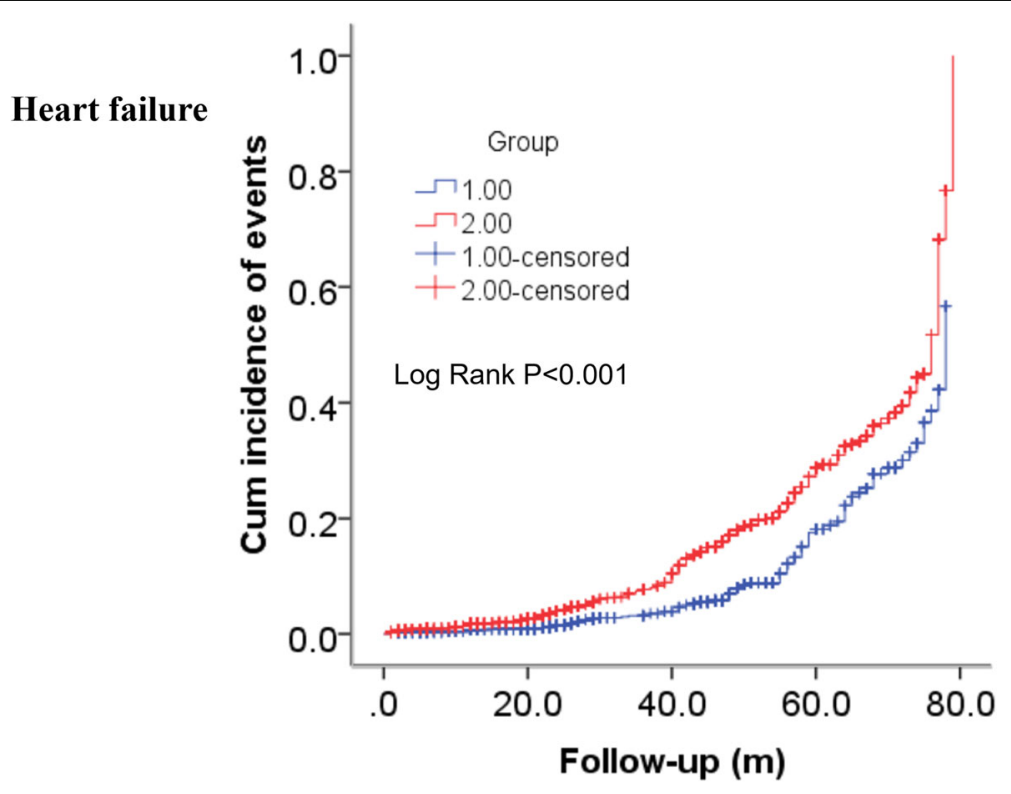

Fig. 3 Cumulative Kaplan-Meier estimates of the time to the first adjudicated occurrence of heart failure 
Table 4 Cox regression analysis results for long-term ACM

\begin{tabular}{lllllll}
\hline Variables & $\mathrm{B}$ & $\mathrm{SE}$ & Wald & $P$-Value & $\mathrm{HR}$ & $95 \% \mathrm{Cl}$ \\
\hline Age & 0.066 & 0.010 & 41.202 & $<\mathbf{0 . 0 0 1}$ & 1.069 & $1.047-1.091$ \\
Gender & -0.175 & 0.256 & 0.466 & 0.495 & 0.840 & $0.508-1.387$ \\
Family history & -1.254 & 0.395 & 10.089 & $\mathbf{0 . 0 0 1}$ & 0.285 & $0.132-0.619$ \\
Hypertension & 0.234 & 0.211 & 1.228 & 0.268 & 1.263 & $0.836-1.909$ \\
Diabetes & 0.582 & 0.208 & 7.823 & $\mathbf{0 . 0 0 5}$ & 1.790 & $1.190-2.692$ \\
Smoking & 0.102 & 0.284 & 0.128 & 0.721 & 1.107 & $0.635-1.930$ \\
Alcohol & 0.323 & 0.313 & 1.066 & 0.302 & 1.381 & $0.748-2.548$ \\
consumption & & & & & & \\
Cr & 0.006 & 0.001 & 31.712 & $<\mathbf{0 . 0 0 1}$ & 1.006 & $1.004-1.008$ \\
UA & 0.001 & 0.001 & 0.427 & 0.513 & 1.001 & $0.999-1.003$ \\
TC & 0.052 & 0.073 & 0.515 & 0.473 & 1.054 & $0.913-1.216$ \\
THR & 0.714 & 0.245 & 8.513 & $\mathbf{0 . 0 0 4}$ & 2.042 & $1.264-3.300$
\end{tabular}

Abbreviation: ACM All-cause mortality, $\mathrm{Cr}$ Creatinine, UA Uric acid, TC Total cholesterol, THR Triglyceride to high-density lipoprotein cholesterol ratio. Note: The boldfaced $P$-Values are statistically different

\section{Study limitations}

In our study, there were several limitations. First, due to a shortage of registered patients, some outcomes were not significantly different, such as CM, MACEs and MACCEs. Second, baseline TG and HDL-C levels were unavailable from a small number of patients enrolled in the CORFCHD-ZZ study, which added to the reduction of the sample size. Third, the follow-up data collection was incomplete. Last but not least, this study was retrospective and aimed to assess the correlation between the THR and long-term mortality in a Chinese patient population with CAD who underwent PCI. Therefore, the findings in our study need to be further demonstrated in different populations.

Table 5 Cox regression analysis results for heart failure

\begin{tabular}{lllllll}
\hline Variables & $\mathrm{B}$ & $\mathrm{SE}$ & Wald & P-Value & $\mathrm{HR}$ & $95 \% \mathrm{Cl}$ \\
\hline Age & 0.002 & 0.004 & 0.266 & 0.606 & 1.002 & $0.994-1.011$ \\
Gender & 0.156 & 0.112 & 1.912 & 0.167 & 1.168 & $0.937-1.456$ \\
Family history & -0.419 & 0.130 & 10.137 & $\mathbf{0 . 0 0 1}$ & 0.658 & $0.510-0.849$ \\
Hypertension & -0.047 & 0.093 & 0.262 & 0.609 & 0.954 & $0.796-1.143$ \\
Diabetes & 0.151 & 0.106 & 2.028 & 0.154 & 1.163 & $0.945-1.432$ \\
Smoking & -0.054 & 0.127 & 0.182 & 0.669 & 0.947 & $0.739-1.214$ \\
Alcohol & -0.217 & 0.156 & 1.943 & 0.163 & 0.805 & $0.593-1.092$ \\
consumption & & & & & & \\
Cr & 0.002 & 0.001 & 5.298 & $\mathbf{0 . 0 2 1}$ & 1.002 & $1.000-1.005$ \\
UA & 0.001 & 0.001 & 0.832 & 0.362 & 1.001 & $0.999-1.002$ \\
TC & -0.250 & 0.046 & 29.633 & $<\mathbf{0 . 0 0 1}$ & 0.779 & $0.712-0.852$ \\
THR & 0.531 & 0.119 & 19.932 & $<\mathbf{0 . 0 0 1}$ & 1.700 & $1.347-2.147$ \\
\hline
\end{tabular}

Abbreviation: Cr Creatinine, UA Uric acid, TC Total cholesterol, THR Triglyceride to high-density lipoprotein cholesterol ratio. Note: The boldfaced $P$-Values are statistically different

\section{Conclusions}

An increased THR was an independent predictor of long-term ACM and heart failure in post-PCI patients with CAD. Moreover, the THR is worth utilizing in clinical practice because it is a precise biomarker that is easily detected at a low cost.

\section{Abbreviations \\ ACM: All-cause mortality; CAD: Coronary artery disease; CIMT: Carotid intima- media thickness; CM: Cardiac mortality; HDL-C: High-density lipoprotein cholesterol; MACCEs: Major adverse cardiac and cerebrovascular events; MACEs: Major adverse cardiac events; PCI: Percutaneous coronary intervention; TG: Triglyceride; THR: Triglyceride to high-density lipoprotein cholesterol ratio}

\section{Acknowledgements}

The authors are grateful to the Department of Cardiology at the First Affiliated Hospital of Zhengzhou University for their help and expertise in conducting this study.

\section{Authors' contributions}

$X Y D$ and YYZ made substantial contributions to study conception and design and to the drafting and critical revision of the manuscript for important intellectual content. JNT and XMY made substantial contributions to the study conception and design and critical revision of the manuscript for important intellectual content. QQG, JCZ, MDC, FHS, ZYL, KW, LZJ, LF, $X T Y, Y B, Z L Z, R J Z$ participated in the collecting and analyzing of the data, writing and reviewing the manuscript. JYZ made substantial contributions to study conception and design, drafting and critical revision of the manuscript for important intellectual content, including study supervision. All authors read and approved the final manuscript.

\section{Funding}

This research was funded by the National Natural Science Foundation of China (81870328, 81760043 and 81800267).

\section{Availability of data and materials}

Due to confidentiality policies, data will not be shared.

\section{Ethics approval and consent to participate}

This study protocol was approved by the ethics committee of the First Affiliated Hospital of Zhengzhou University, and was in line with the Declaration of Helsinki. Due to the retrospective design of the study, the need to obtain informed consent from eligible patients was waived by the ethics committee.

\section{Consent for publication}

Written informed consent for publication was obtained from each author, and there are no other persons who satisfy the criteria for authorship but are not listed.

\section{Competing interests}

The authors declare that they have no competing interests.

\section{Author details}

'Department of Cardiology, First Affiliated Hospital of Zhengzhou University, Zhengzhou 450052, People's Republic of China. ${ }^{2}$ Key Laboratory of Cardiac Injury and Repair of Henan Province, Zhengzhou 450052, People's Republic of China. ${ }^{3}$ Department of Cardiology, The First Affiliated Hospital, and College of Clinical Medicine of Henan University of Science and Technology, Luoyang 471003, People's Republic of China.

Received: 18 August 2019 Accepted: 14 November 2019

Published online: 04 December 2019

\section{References}

1. Lozano R, Naghavi M, Foreman K, et al. Global and regional mortality from 235 causes of death for 20 age groups in 1990 and 2010: a systematic analysis for the global burden of disease study 2010. Lancet. 2012;380(9859): 2095-128. 
2. Kolasińska-Kloch W, Leśniak W, Kieć-Wilk B, et al. Biochemical parameters of endothelial dysfunction in cardiological syndrome X. Scand J Clin Lab Invest. 2002;62(1):7-13.

3. Cosín-Sales J, Pizzi C, Brown S, et al. C-reactive protein, clinical presentation, and ischemic activity in patients with chest pain and normal coronary angiograms. J Am Coll Cardiol. 2003:41(9):1468-74.

4. Kaski JC. Cardiac syndrome $X$ in women: the role of oestrogen deficiency. Heart. 2006;92(Suppl 3):iii5-9.

5. Packard RR, Libby P. Inflammation in atherosclerosis: from vascular biology to biomarker discovery and risk prediction. Clin Chem. 2008;54(1):24-38.

6. Libby P. Current concepts of the pathogenesis of the acute coronary syndromes. Circulation. 2001;104(3):365-72.

7. Reiner Ž. Hypertriglyceridaemia and risk of coronary artery disease. Nat Rev Cardiol. 2017;14(7):401-11.

8. Reiner Ž, De Bacquer D, Kotseva K, et al. Treatment potential for dyslipidaemia management in patients with coronary heart disease across Europe: findings from the EUROASPIRE III survey. Atherosclerosis. 2013; 231(2):300-7.

9. Sarwar N, Danesh J, Eiriksdottir G, et al. Triglycerides and the risk of coronary heart disease: 10,158 incident cases among 262,525 participants in 29 Western prospective studies. Circulation. 2007:115(4):450-8.

10. Barter P, Gotto AM, LaRosa JC, et al. HDL cholesterol, very low levels of LDL cholesterol, and cardiovascular events. N Engl J Med. 2007;357(13):1301-10.

11. Zhou M, Zhu L, Cui $X$, et al. The triglyceride to high-density lipoprotein cholesterol (TG/HDL-C) ratio as a predictor of insulin resistance but not of $\beta$ cell function in a Chinese population with different glucose tolerance status. Lipids Health Dis. 2016;15:104.

12. Gasevic D, Frohlich J, Mancini GJ, et al. Clinical usefulness of lipid ratios to identify men and women with metabolic syndrome: a cross-sectional study. Lipids Health Dis. 2014;13:159.

13. da Luz PL, Favarato D, Faria-Neto JR Jr, et al. High ratio of triglycerides to $\mathrm{HDL}$-cholesterol predicts extensive coronary disease. Clinics (Sao Paulo). 2008;63(4):427-32.

14. Wan $\mathrm{K}$, Zhao J, Huang $\mathrm{H}$, et al. The association between triglyceride/highdensity lipoprotein cholesterol ratio and all-cause mortality in acute coronary syndrome after coronary revascularization. PLoS One. 2015;10(4): e0123521

15. Sultani $\mathrm{R}$, Tong DC, Peverelle $\mathrm{M}$, et al. Elevated Triglycerides to High-Density Lipoprotein Cholesterol (TG/HDL-C) Ratio Predicts Long-Term Mortality in High-Risk Patients. Heart Lung Circ. 2019;(19):30334-8.

16. Weintraub WS, Spertus JA, Kolm P, et al. Effect of PCl on quality of life in patients with stable coronary disease. N Engl J Med. 2008;359(7):677-87.

17. Piccolo R, Giustino G, Mehran R, et al. Stable coronary artery disease: revascularisation and invasive strategies. Lancet. 2015;386(9994):702-13.

18. Aronow WS, Fleg JL, Pepine CJ, et al. ACCF/AHA 2011 expert consensus document on hypertension in the elderly: a report of the American College of Cardiology Foundation task force on clinical expert consensus documents. Circulation. 2011;123(21):2434-506.

19. American Diabetes Association. 2 Classification and Diagnosis of Diabetes: Standards of Medical Care in Diabetes-2018. Diabetes Care. 2018;41(Suppl 1):S13-27.

20. Kim HK, Lee TK. Conditional effects of gain-loss-framed narratives among current smokers at different stages of change. J Health Commun. 2017;22(12):990-8.

21. Cho Y, Kwak S, Lewis SJ, et al. Exploring the utility of alcohol flushing as an instrumental variable for alcohol intake in Koreans. Sci Rep. 2018;8(1):458.

22. Park SJ, Kim YH, Park DW, et al. Randomized trial of stents versus bypass surgery for left main coronary artery disease. N Engl J Med. 2011;364(18):1718-27.

23. Ponikowski P, Voors AA, Anker SD, et al. 2016 ESC guidelines for the diagnosis and treatment of acute and chronic heart failure: the task force for the diagnosis and treatment of acute and chronic heart failure of the European Society of Cardiology (ESC)developed with the special contribution of the heart failure association (HFA) of the ESC. Eur Heart J. 2016;37(27):2129-200.

24. Ndrepepa G, Schuster T, Hadamitzky M, et al. Validation of the bleeding academic research consortium definition of bleeding in patients with coronary artery disease undergoing percutaneous coronary intervention. Circulation. 2012;125(11):1424-31.

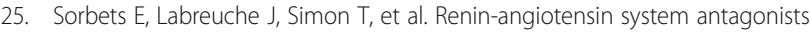
and clinical outcomes in stable coronary artery disease without heart failure. Eur Heart J. 2014;35(26):1760-8
26. Bangalore $S$, Steg $G$, Deedwania $P$, et al. $\beta$-Blocker use and clinical outcomes in stable outpatients with and without coronary artery disease. JAMA. 2012;308(13):1340-9.

27. Laakso M, Kuusisto J. Insulin resistance and hyperglycaemia in cardiovascular disease development. Nat Rev Endocrinol. 2014;10(5):293-302.

28. Kastorini CM, Panagiotakos DB, Georgousopoulou EN, et al. Metabolic syndrome and 10-year cardiovascular disease incidence: the ATTICA study. Nutr Metab Cardiovasc Dis. 2016;26(3):223-31.

29. Li G, Wu HK, Wu XW, et al. Small dense low density lipoprotein-cholesterol and cholesterol ratios to predict arterial stiffness progression in normotensive subjects over a 5-year period. Lipids Health Dis 2018;17(1):27.

30. Pacifico L, Bonci E, Andreoli G, et al. Association of serum triglyceride-toHDL cholesterol ratio with carotid artery intima-media thickness, insulin resistance and nonalcoholic fatty liver disease in children and adolescents. Nutr Metab Cardiovasc Dis. 2014;24(7):737-43.

31. Vasan RS, Short MI, Niiranen TJ, et al. Interrelations between arterial stiffness, target organ damage, and cardiovascular disease outcomes. J Am Heart Assoc. 2019;8(14):e012141.

32. Gepner AD, Young R, Delaney JA, et al. Comparison of coronary artery calcium presence, carotid plaque presence, and carotid intima-media thickness for cardiovascular disease prediction in the Multi-Ethnic Study of Atherosclerosis. Circ Cardiovasc Imaging. 2015:8(1).

33. Avogaro P, Ghiselli G, Soldan S, et al. Relationship of triglycerides and HDL cholesterol in hypertriglyceridemia. Atherosclerosis. 1992;92(1):79-86.

34. Cho Y, Lee SG, Jee SH, et al. Hypertriglyceridemia is a major factor associated with elevated levels of small dense LDL cholesterol in patients with metabolic syndrome. Ann Lab Med. 2015;35(6):586-94.

35. Hsu H, Hsu P, Cheng MH, et al. Lipoprotein subfractions and glucose homeostasis in Prediabetes and diabetes in Taiwan. J Atheroscler Thromb. 2019;26(10):890-914.

36. Maruyama C, Imamura K, Teramoto T. Assessment of LDL particle size by triglyceride/HDL-cholesterol ratio in non-diabetic, healthy subjects without prominent hyperlipidemia. J Atheroscler Thromb. 2003;10(3):186-91.

37. Arsenault BJ, Lemieux I, Després JP, et al. Cholesterol levels in small LDL particles predict the risk of coronary heart disease in the EPIC-Norfolk prospective population study. Eur Heart J. 2007;28(22):2770-7.

\section{Publisher's Note}

Springer Nature remains neutral with regard to jurisdictional claims in published maps and institutional affiliations.
Ready to submit your research? Choose BMC and benefit from:

- fast, convenient online submission

- thorough peer review by experienced researchers in your field

- rapid publication on acceptance

- support for research data, including large and complex data types

- gold Open Access which fosters wider collaboration and increased citations

- maximum visibility for your research: over $100 \mathrm{M}$ website views per year

At $\mathrm{BMC}$, research is always in progress.

Learn more biomedcentral.com/submission 